\title{
Experimentelle Studien über die Funktion des reticuloendothelialen Systems \\ VII. Mitteilung
}

\author{
Einfluss der Chemotherapeutika, besonders der Sulfonamide \\ und Antibiotika auf die Funktion des RES und der Leber \\ Von
}

Schoichi Yamagata, Toshio Aratani, Kiyomi Miura, (山形敞一) (茫谷利夫) (三浦清美)

Kisao Otomo und Chiyoji Hirata

(大交喜佐雄) (平田千代二)

Aus der Med. Klinik von Prof. Dr. T. Kurokawa, Tohoku Universität, Sendai

(Received for publication, February 2, 1945)

Nach der Entdeckung des Salvarsans von Ehrlich ${ }^{1)}$ sind zahlreiche chemotherapeutische Mittel von verschiedenen Forschern wie Domagk, Fleming, ${ }^{3)}$ Waksman usw. ${ }^{4)}$ hergestellt worden. Aber die Meinungen der Autoren gehen auseinander hinsichtlich der Bedeutung des RES bei dem Wirkungsmechanismus der Chemotherapeutika.

Deshalb haben wir in der vorliegenden Arbeit untersucht, welchen Einfluss Chemotherapeutika, besonders Sulfonamide und Antibiotika auf die Funktion des RES und der Leber ausüben. Die Auswahl der Versuchstiere sowie die Methodik war die gleiche wie bei der früheren Mitteilung. ${ }^{5)}$

\section{Versuchsergebnisse}

\section{Einfluss verschiedener Sulfonamide}

Wie aus Tabelle $\mathbf{I}$ zu sehen ist, ruft die intravenöse Injektion von Aktiweis (Sulfanilamide), Trianon (Sulfapyridine), Neogerison (Acetosulfamine), Typhogerison (Acetosulfamine mit Bakterienfiltrat) oder Domian (Sulfapyrimidine), in Menge von $100 \mathrm{mg}$ pro $\mathrm{kg}$, eine Verminderung des Kongorotindex und gleichzeitig eine leichte Vermehrung der Prothrombinzahl hervor. In ähnlicher Weise vermindert sich der Kongorotindex bei intravenöser Injektion von Romezin (Sulfamerazine), Sulzol (Sulfathiazol), Sulzo-S (Sulfaminothiazol), Neodiseptal (Sulfamethylthiazol), Protomin (Promin) oder Thiasin (Sulfaisoxazol), in Menge von $100 \mathrm{mg}$ pro $\mathrm{kg}$, dagegen bleibt die Prothrombinzahl fast unverändert. Aber die Injektion von Theradiazine (Sulfadiazine) im Menge von $100 \mathrm{mg}$ 
TABELLE I

Einfluss verschiedener Sulfonamide

\begin{tabular}{|c|c|c|c|c|c|c|c|}
\hline & \multirow{2}{*}{$\begin{array}{c}\text { Dose } \\
\text { (pro kg) }\end{array}$} & \multirow{2}{*}{ 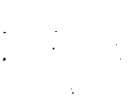 } & \multirow{2}{*}{$\begin{array}{l}\text { Nr. des } \\
\text { Kaninchens }\end{array}$} & \multirow{2}{*}{$\begin{array}{l}\text { Vor } \\
\text { Inj. }\end{array}$} & \multicolumn{3}{|c|}{ Zeit nach Injektion (St.) } \\
\hline & & & & & 1 & 3 & 5 \\
\hline \multirow{2}{*}{ Aktiweis } & \multirow{2}{*}{$\begin{array}{l}100 \mathrm{mg} \\
\text { i.v. }\end{array}$} & K-index & \begin{tabular}{l}
\multicolumn{1}{c}{1} \\
2 \\
Durchschn. \\
Differenz
\end{tabular} & $\begin{array}{l}34.3 \\
33.5 \\
33.9\end{array}$ & $\begin{array}{l}40.2 \\
39.1 \\
39.6 \\
-8.1\end{array}$ & $\begin{array}{r}49.0 \\
47.2 \\
46.9 \\
0.3\end{array}$ & $\begin{array}{l}38.4 \\
46.6 \\
42.6 \\
-1.5\end{array}$ \\
\hline & & P-zahl & $\begin{array}{l}\stackrel{1}{2} \\
\text { Durchschn. } \\
\text { Differenz }\end{array}$ & $\begin{array}{l}1.00 \\
1.00 \\
1.00\end{array}$ & $\begin{array}{l}1.11 \\
1.00 \\
1.06 \\
0.06\end{array}$ & $\begin{array}{l}1.10 \\
1.02 \\
1.06 \\
0.07\end{array}$ & $\begin{array}{l}1.18 \\
1.09 \\
1.14 \\
0.12\end{array}$ \\
\hline \multirow{2}{*}{ Trianon } & \multirow{2}{*}{$\begin{array}{l}100 \mathrm{mg} \\
\text { i.v. }\end{array}$} & $\mathbf{K}$-index & $\begin{array}{l}1 \\
\stackrel{1}{2} \\
\text { Durchschn. } \\
\text { Differenz }\end{array}$ & $\begin{array}{l}52.2 \\
47.8 \\
49.8\end{array}$ & $\begin{array}{r}51.5 \\
52.1 \\
54.8 \\
0.5\end{array}$ & $\begin{array}{l}65.7 \\
53.8 \\
59.4 \\
-1.4\end{array}$ & $\begin{array}{l}58.0 \\
57.7 \\
57.8 \\
-8.5\end{array}$ \\
\hline & & P-zahl & $\begin{array}{l}1 \\
2 \\
\text { Durchschn. } \\
\text { Differenz }\end{array}$ & $\begin{array}{l}1.00 \\
1.00 \\
1.00\end{array}$ & $\begin{array}{l}1.06 \\
1.08 \\
1.07 \\
0.06\end{array}$ & $\begin{array}{l}1.03 \\
1.08 \\
1.06 \\
0.07\end{array}$ & $\begin{array}{l}1.09 \\
1.04 \\
1.07 \\
0.04\end{array}$ \\
\hline \multirow{2}{*}{ Theradiazine } & \multirow{2}{*}{$\begin{array}{l}100 \mathrm{mg} \\
\text { i.v. }\end{array}$} & $\mathrm{K}$-index & \begin{tabular}{l}
\multicolumn{1}{c}{1} \\
$\stackrel{2}{\text { Durchschn. }}$ \\
Differenz
\end{tabular} & $\begin{array}{l}57.2 \\
55.4 \\
56.2\end{array}$ & $\begin{array}{r}67.6 \\
62.1 \\
64.5 \\
3.8\end{array}$ & $\begin{array}{l}59.5 \\
62.4 \\
61.0 \\
-5.2\end{array}$ & $\begin{array}{l}66.6 \\
70.0 \\
68.3 \\
-4.5\end{array}$ \\
\hline & & P-zahl & \begin{tabular}{l}
\multicolumn{1}{c}{1} \\
2 \\
Durchschn. \\
Differenz
\end{tabular} & $\begin{array}{l}1.00 \\
1.00 \\
1.00\end{array}$ & $\begin{array}{r}0.95 \\
0.95 \\
0.95 \\
-0.06\end{array}$ & $\begin{array}{l}1.05 \\
1.01 \\
1.03 \\
0.01\end{array}$ & $\begin{array}{r}0.96 \\
0.97 \\
0.97 \\
-0.07\end{array}$ \\
\hline \multirow{2}{*}{ Romezin } & \multirow{2}{*}{$\begin{array}{l}100 \mathrm{mg} \\
\text { i.v. }\end{array}$} & K-index & \begin{tabular}{l}
\multicolumn{1}{c}{${ }^{2}$} \\
Durchschn. \\
Differenz
\end{tabular} & $\begin{array}{l}54.5 \\
47.1 \\
54.2\end{array}$ & $\begin{array}{l}48.2 \\
54.7 \\
53.7 \\
-5.0\end{array}$ & $\begin{array}{r}66.7 \\
63.2 \\
66.0 \\
0.8\end{array}$ & $\begin{array}{r}66.8 \\
48.4 \\
52.8 \\
-17.9\end{array}$ \\
\hline & & P-zahl & $\begin{array}{c}1 \\
2 \\
\text { Durchschn. } \\
\text { Differenz }\end{array}$ & $\begin{array}{l}1.00 \\
1.00 \\
1.00\end{array}$ & $\begin{array}{l}1.04 \\
0.99 \\
1.02 \\
0.01 \\
\end{array}$ & $\begin{array}{l}1.03 \\
1.05 \\
1.04 \\
0.02 \\
\end{array}$ & $\begin{array}{l}1.11 \\
1.07 \\
1.09 \\
0.05 \\
\end{array}$ \\
\hline \multirow[b]{2}{*}{ Sulzol } & \multirow{2}{*}{$\begin{array}{c}100 \mathrm{mg} \\
\text { i.v. }\end{array}$} & K-index & $\begin{array}{c}1 \\
2 \\
\text { Durchschn. } \\
\text { Differenz }\end{array}$ & $\begin{array}{l}45.1 \\
35.6 \\
40.0\end{array}$ & $\begin{array}{l}47.9 \\
49.3 \\
48.6 \\
-5.2\end{array}$ & $\begin{array}{l}54.1 \\
37.5 \\
45.7 \\
-7.0\end{array}$ & $\begin{array}{l}50.8 \\
42.5 \\
45.9 \\
-4.3\end{array}$ \\
\hline & & P-zahl & $\begin{array}{c}1 \\
2 \\
\text { Durchschn. } \\
\text { Differenz }\end{array}$ & $\begin{array}{l}1.00 \\
1.00 \\
1.00\end{array}$ & $\begin{array}{l}1.05 \\
1.00 \\
1.03 \\
0.03\end{array}$ & $\begin{array}{l}1.01 \\
1.03 \\
1.02 \\
0.03\end{array}$ & $\begin{array}{l}1.06 \\
1.03 \\
1.05 \\
0.03\end{array}$ \\
\hline \multirow{2}{*}{ Neodiseptal } & \multirow{2}{*}{$\begin{array}{l}100 \mathrm{mg} \\
\text { i.v. }\end{array}$} & K-index & $\begin{array}{c}1 \\
2 \\
\text { Durchschn. } \\
\text { Differenz }\end{array}$ & $\begin{array}{l}55.6 \\
52.2 \\
44.7\end{array}$ & $\begin{array}{l}57.5 \\
57.5 \\
46.3 \\
-2.9 \\
\end{array}$ & $\begin{array}{r}58.5 \\
65.7 \\
50.8 \\
-4.9\end{array}$ & $\begin{array}{r}55.2 \\
58.0 \\
44.8 \\
-16.6\end{array}$ \\
\hline & & P-zahl & $\begin{array}{l}1 \\
2 \\
\text { Durchschn. } \\
\text { Differenz }\end{array}$ & $\begin{array}{l}1.00 \\
1.00 \\
1.00\end{array}$ & $\begin{array}{l}1.01 \\
1.09 \\
1.05 \\
0.04\end{array}$ & $\begin{array}{l}1.03 \\
1.07 \\
1.05 \\
0.03\end{array}$ & $\begin{array}{l}1.11 \\
1.04 \\
1.08 \\
0.04\end{array}$ \\
\hline
\end{tabular}


Einfluss der Chemotherapeutika auf RES u. Leber

\begin{tabular}{|c|c|c|c|c|c|c|c|}
\hline \multirow{2}{*}{ Sulzol-S } & \multirow{2}{*}{$\begin{array}{l}100 \mathrm{mg} \\
\text { i. } \mathrm{v} .\end{array}$} & $\mathrm{K}$-index & \begin{tabular}{l}
\multicolumn{1}{c}{1} \\
\multicolumn{2}{|c}{} \\
Durchschn. \\
Differenz
\end{tabular} & $\begin{array}{l}61.0 \\
50.0 \\
55.9\end{array}$ & $\begin{array}{r}54.8 \\
34.5 \\
44.8 \\
-14.3\end{array}$ & $\begin{array}{r}54.5 \\
42.8 \\
49.5 \\
-10.7\end{array}$ & $\begin{array}{l}60.0 \\
42.8 \\
52.8 \\
-6.8\end{array}$ \\
\hline & & P-zahl & $\begin{array}{l}\quad 1 \\
\quad 2 \\
\text { Durchschn. } \\
\text { Differenz }\end{array}$ & $\begin{array}{l}1.00 \\
1.00 \\
1.00\end{array}$ & $\begin{array}{l}1.03 \\
1.03 \\
1.03 \\
0.02\end{array}$ & $\begin{array}{r}1.04 \\
1.00 \\
1.02 \\
-0.01\end{array}$ & $\begin{array}{c}1.04 \\
1.02 \\
1.03 \\
0\end{array}$ \\
\hline \multirow{2}{*}{ Neogerison } & \multirow{2}{*}{$\begin{array}{l}100 \mathrm{mg} \\
\text { i.v. }\end{array}$} & K-index & \begin{tabular}{l}
$\quad 1$ \\
\multicolumn{2}{c}{1} \\
Durchschn. \\
Differenz
\end{tabular} & $\begin{array}{l}58.2 \\
39.4 \\
49.1\end{array}$ & $\begin{array}{r}54.0 \\
44.3 \\
49.3 \\
-11.6\end{array}$ & $\begin{array}{l}72.4 \\
54.8 \\
64.7 \\
-3.5\end{array}$ & $\begin{array}{r}69.5 \\
43.3 \\
56.0 \\
-10.6\end{array}$ \\
\hline & & P-zahl & \begin{tabular}{l}
1 \\
\multicolumn{2}{c}{} \\
Durchschn. \\
Differenz
\end{tabular} & $\begin{array}{l}1.00 \\
1.00 \\
1.00\end{array}$ & $\begin{array}{l}1.01 \\
1.09 \\
1.05 \\
0.06\end{array}$ & $\begin{array}{l}1.03 \\
1.07 \\
1.05 \\
0.08\end{array}$ & $\begin{array}{l}1.11 \\
1.04 \\
1.08 \\
0.10\end{array}$ \\
\hline \multirow[t]{2}{*}{ Typhogerison } & \multirow{2}{*}{$\begin{array}{l}100 \mathrm{mg} \\
\text { i.v. }\end{array}$} & $\mathrm{K}$-index & $\begin{array}{l}1 \\
2 \\
\text { Durchschn. } \\
\text { Differenz }\end{array}$ & $\begin{array}{l}37.6 \\
36.0 \\
37.1\end{array}$ & $\begin{array}{r}37.6 \\
43.0 \\
40.3 \\
-10.6\end{array}$ & $\begin{array}{l}47.7 \\
51.3 \\
49.3 \\
-0.5\end{array}$ & $\begin{array}{r}52.9 \\
56.7 \\
55.4 \\
8.1\end{array}$ \\
\hline & & P-zahl & \begin{tabular}{l}
\multicolumn{1}{c}{1} \\
2 \\
Durchschn. \\
Differenz
\end{tabular} & $\begin{array}{l}1.00 \\
1.00 \\
1.00\end{array}$ & $\begin{array}{l}1.11 \\
1.08 \\
1.10 \\
0.10\end{array}$ & $\begin{array}{l}1.16 \\
1.08 \\
1.12 \\
0.09\end{array}$ & $\begin{array}{l}1.18 \\
1.07 \\
1.13 \\
0.11\end{array}$ \\
\hline \multirow[t]{2}{*}{ Protomin } & \multirow{2}{*}{$\begin{array}{l}100 \mathrm{mg} \\
\text { i.v. }\end{array}$} & $\mathrm{K}$-index & \begin{tabular}{l}
$\quad 1$ \\
\multicolumn{2}{c}{1} \\
Durchschn. \\
Differenz
\end{tabular} & $\begin{array}{l}59.2 \\
35.0 \\
48.2\end{array}$ & $\begin{array}{r}56.0 \\
33.4 \\
46.8 \\
-15.2 \\
\end{array}$ & $\begin{array}{l}59.2 \\
40.9 \\
56.7 \\
-4.2 \\
\end{array}$ & $\begin{array}{l}60.0 \\
42.1 \\
52.3 \\
-6.1\end{array}$ \\
\hline & & P-zahl & $\begin{array}{l}1 \\
1 \\
2 \\
\text { Durchschn. } \\
\text { Differenz } \\
\end{array}$ & $\begin{array}{l}1.00 \\
1.00 \\
1.00\end{array}$ & $\begin{array}{l}1.07 \\
1.01 \\
1.04 \\
0.04 \\
\end{array}$ & $\begin{array}{l}1.02 \\
0.97 \\
1.00 \\
0.01 \\
\end{array}$ & $\begin{array}{c}0.98 \\
1.06 \\
1.02 \\
0 \\
\end{array}$ \\
\hline \multirow{2}{*}{ Homosulfamin } & \multirow{2}{*}{$\begin{array}{c}100 \mathrm{mg} \\
\text { i.v. }\end{array}$} & K-index & $\begin{array}{l}1 \\
2 \\
\text { Durchschn. } \\
\text { Differenz }\end{array}$ & $\begin{array}{l}50.2 \\
31.0 \\
40.6\end{array}$ & $\begin{array}{r}54.8 \\
39.9 \\
47.7 \\
2.6 \\
\end{array}$ & $\begin{array}{r}63.0 \\
46.2 \\
56.2 \\
4.6 \\
\end{array}$ & $\begin{array}{r}69.6 \\
51.0 \\
62.6 \\
5.5 \\
\end{array}$ \\
\hline & & P-zahl & \begin{tabular}{l}
\multicolumn{1}{c}{1} \\
2 \\
Durchschn. \\
Differenz
\end{tabular} & $\begin{array}{l}1.00 \\
1.00 \\
1.00\end{array}$ & $\begin{array}{l}1.14 \\
1.12 \\
1.13 \\
0.12 \\
\end{array}$ & $\begin{array}{l}1.08 \\
1.11 \\
1.10 \\
0.10 \\
\end{array}$ & $\begin{array}{c}1.07 \\
1.01 \\
1.04 \\
0 \\
\end{array}$ \\
\hline \multirow{2}{*}{ Thiasin } & \multirow{2}{*}{$\begin{array}{l}100 \mathrm{mg} \\
\text { i.v. }\end{array}$} & $\mathrm{K}$-index & \begin{tabular}{l}
\multicolumn{1}{c}{1} \\
2 \\
Durchschn. \\
Differenz
\end{tabular} & $\begin{array}{l}73.8 \\
71.7 \\
72.8\end{array}$ & $\begin{array}{r}67.8 \\
64.8 \\
66.7 \\
-17.9 \\
\end{array}$ & $\begin{array}{r}60.8 \\
73.7 \\
66.2 \\
-25.7 \\
\end{array}$ & $\begin{array}{r}72.8 \\
76.7 \\
74.4 \\
-15.9 \\
\end{array}$ \\
\hline & & P-zahl & $\begin{array}{l}1 \\
2 \\
\text { Durchschn. } \\
\text { Differenz }\end{array}$ & $\begin{array}{l}1.00 \\
1.00 \\
1.00\end{array}$ & $\begin{array}{r}0.98 \\
0.98 \\
0.98 \\
-0.01 \\
\end{array}$ & $\begin{array}{l}0.99 \\
0.98 \\
0.99 \\
0.02 \\
\end{array}$ & $\begin{array}{l}1.00 \\
1.00 \\
1.00 \\
0.02 \\
\end{array}$ \\
\hline \multirow[b]{2}{*}{ Domian } & \multirow{2}{*}{$\begin{array}{l}100 \mathrm{mg} \\
\text { i.v. }\end{array}$} & $\mathrm{K}$-index & $\begin{array}{c}1 \\
\frac{1}{2} \\
\text { Durchschn. } \\
\text { Differenz } \\
1\end{array}$ & $\begin{array}{l}52.8 \\
49.5 \\
51.1\end{array}$ & $\begin{array}{r}42.6 \\
48.2 \\
45.5 \\
-17.4 \\
\end{array}$ & $\begin{array}{l}61.9 \\
63.1 \\
62.5 \\
-7.7 \\
\end{array}$ & $\begin{array}{r}50.0 \\
60.3 \\
54.3 \\
-14.3 \\
\end{array}$ \\
\hline & & P-zahl & \begin{tabular}{l}
1 \\
\multicolumn{2}{c}{2} \\
Durchschn. \\
Differenz
\end{tabular} & $\begin{array}{l}1.00 \\
1.00 \\
1.00\end{array}$ & $\begin{array}{l}1.07 \\
1.06 \\
1.07 \\
0.08\end{array}$ & $\begin{array}{l}1.07 \\
1.05 \\
1.06 \\
0.09\end{array}$ & $\begin{array}{l}1.01 \\
1.05 \\
1.03 \\
0.05\end{array}$ \\
\hline
\end{tabular}


pro kg setzt den Kongorotindex sowie die Prothrombinzahl herab, im Gegensatz dazu ruft die Injektion von Homosulfamin (Marfanil) eine Vermehrung des Kongorotindex und der Prothrombinzahl hervor.

Daraus ist ersichtlich, dass diese Sulfonamide, nicht aber Homosulfamin, die Funktion des RES steigern, dagegen wird diejenige der Leberzellen nur durch Aktiweis, Trianon, Neogerison, Typhogerison, Domian und Homosulfamin erhöht.

\section{Einfluss der Antibiotika}

Wenn man Penicillin G, Mix oder K, in Menge von $20000 \mathrm{EH}$ intramuskulär injiziert, so vermindert sich, wie Tabelle II zeigt, der Kongorotindex und die Prothrombinzahl vermehrt sich in leichtem Grade. Aber der Kongorotindex vermindert sich und die Prothrombinzahl bleibt unverändert durch wässeriges Penicillin $G$ in Menge von $30000 \mathrm{EH}$, während der Kongorotindex bei intramuskulärer Injektion des Penicillins $\mathrm{K}$ in Menge von $10000 \mathrm{EH}$ oder des öligen Penicillins $\mathrm{G}$ in Menge von $30000 \mathrm{EH}$ unverändert bleibt.

Aus Tabelle III sieht man, dass die Injektion von Streptomycin in Menge von $20000 \mathrm{EH}$, ja sogar $5000 \mathrm{EH}$ den Kongorotindex herabsetzt, doch erhöht sie die Prothrombinzahl. Aber die Injektion von Colistin in Menge von $100000 \mathrm{EH}$ verursacht eine Verminderung des Kongorotindex sowie der Prothrombinzahl.

Deshalb ist es klar, dass diese Antibiotika, nicht aber Penicillin in kleiner Menge und öligem Penicillin, die Funktion des RES ziemlich auffallend, aber diejenige der Leberzellen nur leichtgradig steigern.

3. Einfluss der Tuberkulostatika

Wie schon erwähnt, steigert das als Tuberkulostatika ausgezeichnete Streptomycin und Protomin die Funktion des RES. Tabelle IV zeigt, dass die subkutane Injektion von PAS (Paraaminosalicylsäure) in Menge von $200 \mathrm{mg}$ pro $\mathrm{kg}$ den Kongorotindex erhöht, aber in Menge von 33 $\mathrm{mg}$ pro $\mathrm{kg}$ denselben herabsetzt, während er durch PAS in Menge von $50 \mathrm{mg}$ pro $\mathrm{kg}$ fast unverändert bleibt. Weiterhin bemerken wir, dass die subkutane Injektion von Tibione (Thiosemicarbazone) in Menge von $10 \mathrm{mg}$ pro kg den Kongorotindex auffällig herabsetzt, während derselbe durch Tibione in Menge von $50 \mathrm{mg}$ pro $\mathrm{kg}$ fast unbeeinflusst bleibt. Aber die Prothrombinzahl verändert sich dabei keinesfalls. Wenn man Cepharantin, das von Hasegawa ${ }^{6}$ ) als Tuberkulostatika empfohlene Mittel, in Menge von $0.05 \mathrm{mg}$ pro kg injiziert, vermindert sich der Kongorotindex und vermehrt sich die Prothrombinzahl in mässigem Grade.

Aus den obigen Versuchen wird klar, dass diese antituberkulösen Mittle in geeigneter Menge die Funktion des RES steigern, aber nicht die Leberzellenfunktion, mit Ausnahme von Cepharantin.

4. Einfluss der antisyphilitischen Mittel 
TABELLE II

Einfluss des Penicillins

\begin{tabular}{|c|c|c|c|c|c|c|c|}
\hline & \multirow{2}{*}{$\begin{array}{c}\text { Dose } \\
\text { (pro kg) }\end{array}$} & & \multirow{2}{*}{$\begin{array}{l}\text { Nr. des } \\
\text { Kaninchens }\end{array}$} & \multirow{2}{*}{$\begin{array}{l}\text { Vor } \\
\text { Inj. }\end{array}$} & \multicolumn{3}{|c|}{ Zeit nach Injektion (St.) } \\
\hline & & & & & 1 & 3 & 5 \\
\hline \multirow{2}{*}{ Penicillin G } & \multirow{2}{*}{$\begin{array}{l}20000 \mathrm{EH} \\
\text { i.m. }\end{array}$} & $\mathbf{K}$-index & $\begin{array}{c}1 \\
2 \\
\text { Durchschn. } \\
\text { Differenz }\end{array}$ & $\begin{array}{l}54.1 \\
53.6 \\
53.9\end{array}$ & $\begin{array}{r}55.4 \\
57.2 \\
56.2 \\
-11.5 \\
\end{array}$ & $\begin{array}{l}59.2 \\
56.3 \\
57.9 \\
-8.7 \\
\end{array}$ & $\begin{array}{l}61.8 \\
53.1 \\
59.0 \\
-5.1\end{array}$ \\
\hline & & P-zahl & $\begin{array}{l}1 \\
2 \\
\text { Durchschn. } \\
\text { Differenz }\end{array}$ & $\begin{array}{l}1.00 \\
1.00 \\
1.00\end{array}$ & $\begin{array}{l}1.03 \\
1.02 \\
1.03 \\
0.03\end{array}$ & $\begin{array}{l}1.11 \\
1.03 \\
1.07 \\
0.08\end{array}$ & $\begin{array}{l}1.16 \\
1.09 \\
1.13 \\
0.11\end{array}$ \\
\hline \multirow{2}{*}{$\begin{array}{l}\text { Penicillin } \\
\text { Mix }\end{array}$} & \multirow{2}{*}{$\begin{array}{l}20000 \mathrm{EH} \\
\text { i.m. }\end{array}$} & $\mathrm{K}$-index & $\begin{array}{c}1 \\
2 \\
\text { Durchschn. } \\
\text { Differenz }\end{array}$ & $\begin{array}{l}45.9 \\
43.9 \\
44.9\end{array}$ & $\begin{array}{r}43.3 \\
48.0 \\
45.4 \\
-13.3 \\
\end{array}$ & $\begin{array}{l}51.5 \\
56.0 \\
53.6 \\
-3.4 \\
\end{array}$ & $\begin{array}{l}54.6 \\
51.5 \\
52.9 \\
-2.2 \\
\end{array}$ \\
\hline & & P-zahl & $\begin{array}{c}1 \\
2 \\
\text { Durchschn. } \\
\text { Differenz }\end{array}$ & $\begin{array}{l}1.00 \\
1.00 \\
1.00\end{array}$ & $\begin{array}{l}1.10 \\
1.02 \\
1.06 \\
0.06 \\
\end{array}$ & $\begin{array}{l}1.04 \\
1.09 \\
1.07 \\
0.08\end{array}$ & $\begin{array}{l}1.08 \\
1.02 \\
1.05 \\
0.03\end{array}$ \\
\hline \multirow{4}{*}{ Penicillin $\mathrm{K}$} & \multirow{2}{*}{$\begin{array}{l}20000 \mathrm{EH} \\
\text { i.m. }\end{array}$} & $\mathrm{K}$-index & $\begin{array}{l}1 \\
2 \\
\text { Durchschn. } \\
\text { Differenz }\end{array}$ & $\begin{array}{l}42.7 \\
28.9 \\
36.7\end{array}$ & $\begin{array}{r}45.0 \\
33.1 \\
39.9 \\
-10.6\end{array}$ & $\begin{array}{l}48.0 \\
39.0 \\
44.0 \\
-5.4\end{array}$ & $\begin{array}{l}66.7 \\
44.1 \\
57.2 \\
10.3\end{array}$ \\
\hline & & P-zahl & $\begin{array}{c}1 \\
2 \\
\text { Durchschn. } \\
\text { Differenz }\end{array}$ & $\begin{array}{l}1.00 \\
1.00 \\
1.00\end{array}$ & $\begin{array}{l}1.01 \\
1.02 \\
1.02 \\
0.02\end{array}$ & $\begin{array}{l}1.06 \\
1.04 \\
1.04 \\
0.05\end{array}$ & $\begin{array}{r}0.99 \\
0.99 \\
0.99 \\
-0.03\end{array}$ \\
\hline & \multirow{2}{*}{$\begin{array}{l}10000 \mathrm{EH} \\
\text { i.m. }\end{array}$} & $\mathrm{K}$-index & $\begin{array}{c}1 \\
2 \\
\text { Durchschn. } \\
\text { Differenz }\end{array}$ & $\begin{array}{l}52.6 \\
45.1 \\
48.8\end{array}$ & $\begin{array}{r}60.0 \\
51.5 \\
55.8 \\
2.5 \\
\end{array}$ & $\begin{array}{r}62.3 \\
53.0 \\
58.0 \\
-1.8 \\
\end{array}$ & $\begin{array}{r}70.6 \\
67.6 \\
69.3 \\
4.0 \\
\end{array}$ \\
\hline & & P-zahI & $\begin{array}{l}1 \\
2 \\
\text { Durchscun. } \\
\text { Differenz }\end{array}$ & $\begin{array}{l}1.00 \\
1.00 \\
1.00\end{array}$ & $\begin{array}{l}1.08 \\
1.00 \\
1.04 \\
0.03\end{array}$ & $\begin{array}{l}1.08 \\
1.13 \\
1.11 \\
0.09\end{array}$ & $\begin{array}{l}1.18 \\
1.02 \\
1.10 \\
0.06\end{array}$ \\
\hline \multirow{2}{*}{$\begin{array}{l}\text { Oeliges } \\
\text { Penicillin G }\end{array}$} & \multirow{2}{*}{$\begin{array}{l}30000 \mathrm{EH} \\
\text { i.m. }\end{array}$} & K-index & $\begin{array}{l}1 \\
2 \\
\text { Durchschn. } \\
\text { Differenz }\end{array}$ & $\begin{array}{l}48.7 \\
48.7 \\
48.7\end{array}$ & $\begin{array}{r}67.8 \\
57.2 \\
62.6 \\
0.1\end{array}$ & $\begin{array}{r}70.8 \\
56.5 \\
63.7 \\
2.3\end{array}$ & $\begin{array}{r}70.0 \\
64.1 \\
67.1 \\
8.2\end{array}$ \\
\hline & & P-zahl & \begin{tabular}{l}
\multicolumn{1}{c}{} \\
2 \\
Durchschn. \\
Differenz
\end{tabular} & $\begin{array}{l}1.00 \\
1.00 \\
1.00\end{array}$ & $\begin{array}{l}1.03 \\
1.03 \\
1.03 \\
0.03\end{array}$ & $\begin{array}{l}1.16 \\
1.02 \\
1.09 \\
0.10\end{array}$ & $\begin{array}{l}1.14 \\
1.03 \\
1.09 \\
0.07\end{array}$ \\
\hline \multirow{2}{*}{$\begin{array}{l}\text { Wassriges } \\
\text { Penicillin G }\end{array}$} & \multirow{2}{*}{$\begin{array}{l}30000 \mathrm{EH} \\
\text { i.m. }\end{array}$} & $\mathrm{K}$-index & $\begin{array}{l}1 \\
2 \\
\text { Durchschn. } \\
\text { Differenz }\end{array}$ & $\begin{array}{l}40.5 \\
26.7 \\
34.0\end{array}$ & $\begin{array}{r}40.8 \\
24.7 \\
33.5 \\
-14.3 \\
\end{array}$ & $\begin{array}{r}30.7 \\
25.8 \\
28.1 \\
-18.6\end{array}$ & $\begin{array}{r}34.5 \\
30.4 \\
32.5 \\
-11.7 \\
\end{array}$ \\
\hline & & P-zahl & \begin{tabular}{l}
\multicolumn{1}{c}{1} \\
2 \\
Durchschn. \\
Differenz
\end{tabular} & $\begin{array}{l}1.00 \\
1.00 \\
1.00\end{array}$ & $\begin{array}{l}1.01 \\
1.00 \\
1.01 \\
0.01\end{array}$ & $\begin{array}{l}1.04 \\
1.02 \\
1.03 \\
0.04\end{array}$ & $\begin{array}{r}1.00 \\
1.00 \\
1.00 \\
-0.02\end{array}$ \\
\hline
\end{tabular}

Wie aus Tabelle $\mathrm{V}$ zu ersehen ist, erhöht die intravenöse Injektion von Mapharsol (Mapharsene), den antisyphilitischen Arsenpräparaten, in Menge von $5 \mathrm{mg}$ pro $\mathrm{kg}$ den Kongorotindex, dagegen setzt dieselbe Injektion in Menge von $0.5 \mathrm{mg}$ pro $\mathrm{kg}$ den 'Kongorotindex herab, indem 
TABELLE III

Einfluss anderer Antibiotika

\begin{tabular}{|c|c|c|c|c|c|c|c|}
\hline & \multirow{2}{*}{$\begin{array}{c}\text { Dose } \\
\text { (pro kg) }\end{array}$} & & \multirow{2}{*}{$\begin{array}{l}\text { Nr. des } \\
\text { Kaninchens }\end{array}$} & \multirow{2}{*}{$\begin{array}{l}\text { Vor } \\
\text { Inj. }\end{array}$} & \multicolumn{3}{|c|}{ Zeit nach Injektion (St.) } \\
\hline & & & & & 1 & 3 & 5 \\
\hline \multirow{4}{*}{ Streptomycin } & \multirow{2}{*}{$\begin{array}{c}20000 \mathrm{EH} \\
\text { i.m. }\end{array}$} & $\mathbf{K}$-index & \begin{tabular}{l}
\multicolumn{1}{c}{$\stackrel{1}{2}$} \\
Durchschn. \\
Differenz
\end{tabular} & $\begin{array}{l}42.6 \\
41.9 \\
42.3\end{array}$ & $\begin{array}{l}45.5 \\
55.2 \\
50.0 \\
-6.1\end{array}$ & $\begin{array}{l}33.9 \\
52.7 \\
45.3 \\
-9.4\end{array}$ & $\begin{array}{l}47.7 \\
57.0 \\
52.3 \\
-0.2\end{array}$ \\
\hline & & P-zahl & $\begin{array}{l}\stackrel{1}{2} \\
\text { Durchschn. } \\
\text { Differenz }\end{array}$ & $\begin{array}{l}1.00 \\
1.00 \\
1.00\end{array}$ & $\begin{array}{l}1.01 \\
1.04 \\
1.03 \\
0.03\end{array}$ & $\begin{array}{l}1.05 \\
1.07 \\
1.06 \\
0.07\end{array}$ & $\begin{array}{l}1.09 \\
1.06 \\
1.08 \\
0.06\end{array}$ \\
\hline & \multirow{2}{*}{$\begin{array}{l}5000 \mathrm{EH} \\
\text { i.m. }\end{array}$} & K-index & $\begin{array}{l}1 \\
\stackrel{1}{2} \\
\text { Durchschn. } \\
\text { Differenz }\end{array}$ & $\begin{array}{l}47.0 \\
41.6 \\
44.3\end{array}$ & $\begin{array}{l}53.2 \\
45.3 \\
49.6 \\
-8.5\end{array}$ & $\begin{array}{l}57.5 \\
56.0 \\
56.8 \\
-0.2\end{array}$ & $\begin{array}{l}52.2 \\
53.2 \\
52.5 \\
-2.4\end{array}$ \\
\hline & & P-zahl & $\begin{array}{l}1 \\
2 \\
\text { Durchschn. } \\
\text { Differenz }\end{array}$ & $\begin{array}{l}1.00 \\
1.00 \\
1.00\end{array}$ & $\begin{array}{c}1.03 \\
0.97 \\
1.00 \\
0\end{array}$ & $\begin{array}{l}1.10 \\
1.07 \\
1.09 \\
0.10\end{array}$ & $\begin{array}{l}1.05 \\
1.06 \\
1.06 \\
0.04\end{array}$ \\
\hline \multirow{2}{*}{ Colistin } & \multirow{2}{*}{$\begin{array}{c}100000 \mathrm{EH} \\
\text { i.m. }\end{array}$} & $\mathrm{K}$-index & \begin{tabular}{l}
\multicolumn{1}{c}{1} \\
2 \\
Durchschn. \\
Differenz
\end{tabular} & $\begin{array}{l}50.0 \\
25.0 \\
37.5\end{array}$ & $\begin{array}{r}45.3 \\
30.5 \\
38.1 \\
-13.2 \\
\end{array}$ & $\begin{array}{l}47.8 \\
35.1 \\
41.6 \\
-8.6\end{array}$ & $\begin{array}{r}58.8 \\
50.0 \\
54.3 \\
6.6\end{array}$ \\
\hline & & P-zahl & $\begin{array}{l}1 \\
\quad 2 \\
\text { Durchschn. } \\
\text { Differenz }\end{array}$ & $\begin{array}{l}1.00 \\
1.00 \\
1.00\end{array}$ & $\begin{array}{l}1.03 \\
1.01 \\
1.02 \\
0.02\end{array}$ & $\begin{array}{c}0.95 \\
1.02 \\
0.99 \\
0\end{array}$ & $\begin{array}{l}1.05 \\
1.01 \\
1.03 \\
0.01\end{array}$ \\
\hline
\end{tabular}

sie die Prothrombinzahl erhöht. Wenn man aber Neoneonatvarsan (Neosalvarsan) in Maenge von $0.5 \mathrm{mg}$ pro $\mathrm{kg}$ intravenös injiziert, so ist eine Verminderung des Kongorotindex sehr gering, auch bleibt die Prothrombinzahl fast unverändert.

Daraus wird klar, dass diese Arsenpräparate in kleiner Menge die Funktion des RES steigern. tika

5. Einfluss des Luminals auf die Wirkung der Sulfonamide und Antibio-

Aus Tabelle VI ersieht man, dass die oben erwähnte Verminderung des Kongorotindex nach der Injektion von Neogerison, Penicillin $\mathrm{K}$ oder Streptomycin durch Luminal fast völlig gehemmt wird, wobei eine Veränderung der Prothrombinzahl nicht einheitlich ist.

Also ist zu vermuten, dass diese Sulfonamide und Antibiotika vermittelst des vegetativen Nervenzentrums in ähnlicher Weise wie bei Vitamin die Funktion des RES stimulieren.

6. Einfluss der Antibiotika auf die Phagozytose der Leukozyten 
TABELLE IV

Einfluss der Tuberkulostatika

\begin{tabular}{|c|c|c|c|c|c|c|c|}
\hline & \multirow{2}{*}{$\begin{array}{c}\text { Dose } \\
\text { (pro kg) }\end{array}$} & & \multirow{2}{*}{$\begin{array}{l}\text { Nr. des } \\
\text { Kaninchens }\end{array}$} & \multirow{2}{*}{$\begin{array}{l}\text { Vor } \\
\text { Inj. }\end{array}$} & \multicolumn{3}{|c|}{ Zeit nach Injektion (St.) } \\
\hline & & & & & 1 & 3 & 5 \\
\hline \multirow{6}{*}{ PAS } & \multirow{2}{*}{$\begin{array}{l}200 \mathrm{mg} \\
\text { s.c. }\end{array}$} & $\mathrm{K}$-index & $\begin{array}{l}1 \\
2 \\
\text { Durchschn. } \\
\text { Differenz }\end{array}$ & $\begin{array}{l}53.5 \\
44.7 \\
48.6\end{array}$ & $\begin{array}{l}80.8 \\
57.3 \\
68.2 \\
16.4 \\
\end{array}$ & $\begin{array}{l}62.8 \\
66.7 \\
64.9 \\
12.0 \\
\end{array}$ & $\begin{array}{l}61.3 \\
76.0 \\
68.4 \\
16.1 \\
\end{array}$ \\
\hline & & P-zahl & $\begin{array}{l}1 \\
2 \\
\text { Durchschn. } \\
\text { Differenz }\end{array}$ & $\begin{array}{l}1.00 \\
1.00 \\
1.00\end{array}$ & $\begin{array}{c}1.02 \\
0.99 \\
1.01 \\
0 \\
\end{array}$ & $\begin{array}{c}1.04 \\
1.01 \\
1.03 \\
0 \\
\end{array}$ & $\begin{array}{r}1.02 \\
1.01 \\
1.02 \\
-0.01 \\
\end{array}$ \\
\hline & \multirow{2}{*}{$\begin{array}{l}50 \mathrm{mg} \\
\text { s.c. }\end{array}$} & $\mathrm{K}$-index & $\begin{array}{l}1 \\
2 \\
\text { Durchschn. } \\
\text { Differenz }\end{array}$ & $\begin{array}{l}44.1 \\
34.6 \\
39.3\end{array}$ & $\begin{array}{r}44.7 \\
43.2 \\
43.9 \\
0.2 \\
\end{array}$ & $\begin{array}{r}46.5 \\
45.8 \\
45.6 \\
2.0 \\
\end{array}$ & $\begin{array}{r}44.3 \\
44.1 \\
44.3 \\
1.3 \\
\end{array}$ \\
\hline & & P-zahl & $\begin{array}{l}1 \\
1 \\
\text { Durchschn. } \\
\text { Differenz }\end{array}$ & $\begin{array}{l}1.00 \\
1.00 \\
1.00\end{array}$ & $\begin{array}{r}0.98 \\
1.00 \\
0.99 \\
-0.02 \\
\end{array}$ & $\begin{array}{r}0.93 \\
1.00 \\
0.97 \\
-0.06\end{array}$ & $\begin{array}{r}1.03 \\
1.00 \\
1.02 \\
-0.01 \\
\end{array}$ \\
\hline & \multirow{2}{*}{$\begin{array}{l}33 \mathrm{mg} \\
\text { s.c. }\end{array}$} & K-index & $\begin{array}{l}1 \\
2 \\
\text { Durchschn. } \\
\text { Differenz }\end{array}$ & $\begin{array}{l}65.7 \\
61.1 \\
63.6\end{array}$ & $\begin{array}{r}43.9 \\
40.8 \\
42.2 \\
-24.6\end{array}$ & $\begin{array}{r}65.7 \\
48.3 \\
57.1 \\
-10.8\end{array}$ & $\begin{array}{r}71.8 \\
70.0 \\
71.1 \\
3.8\end{array}$ \\
\hline & & P-zahl. & $\begin{array}{l}1 \\
2 \\
\text { Durchschn. } \\
\text { Differenz }\end{array}$ & $\begin{array}{l}1.00 \\
1.00 \\
1.00\end{array}$ & $\begin{array}{c}1.01 \\
1.00 \\
1.01 \\
0 \\
\end{array}$ & $\begin{array}{r}1.02 \\
1.00 \\
1.01 \\
-0.02 \\
\end{array}$ & $\begin{array}{c}.04 \\
1.02 \\
1.03 \\
0 \\
\end{array}$ \\
\hline \multirow{4}{*}{ Tibione } & \multirow{2}{*}{$\begin{array}{l}50 \mathrm{mg} \\
\text { s.c. }\end{array}$} & $\mathrm{K}$-index & $\begin{array}{l}1 \\
\quad 1 \\
\text { Durchschn. } \\
\text { Differenz }\end{array}$ & $\begin{array}{l}48.0 \\
42.9 \\
45.6\end{array}$ & $\begin{array}{r}74.0 \\
53.2 \\
63.7 \\
7.1 \\
\end{array}$ & $\begin{array}{r}77.3 \\
63.2 \\
70.2 \\
5.5 \\
\end{array}$ & $\begin{array}{l}63.5 \\
51.8 \\
57.7 \\
-5.4 \\
\end{array}$ \\
\hline & & P-zahl & $\begin{array}{l}1 \\
2 \\
\text { Durchschn. } \\
\text { Differenz }\end{array}$ & $\begin{array}{l}1.00 \\
1.00 \\
1.00\end{array}$ & $\begin{array}{r}0.96 \\
0.92 \\
0.94 \\
-0.05 \\
\end{array}$ & $\begin{array}{r}0.91 \\
0.89 \\
0.90 \\
-0.07 \\
\end{array}$ & $\begin{array}{r}0.91 \\
0.89 \\
0.90 \\
-0.08 \\
\end{array}$ \\
\hline & \multirow{2}{*}{$\begin{array}{l}10 \mathrm{mg} \\
\text { s.c. }\end{array}$} & $\mathrm{K}$-index & $\begin{array}{c}1 \\
2 \\
\text { Durchschn. } \\
\text { Differenz }\end{array}$ & $\begin{array}{l}61.3 \\
46.8 \\
54.3\end{array}$ & $\begin{array}{r}44.7 \\
44.8 \\
42.7 \\
-13.4 \\
\end{array}$ & $\begin{array}{r}60.8 \\
51.1 \\
54.0 \\
-19.4 \\
\end{array}$ & $\begin{array}{r}65.3 \\
56.3 \\
61.2 \\
-10.6\end{array}$ \\
\hline & & P-zahl & $\begin{array}{l}1 \\
2 \\
\text { Durchschn. } \\
\text { Differenz }\end{array}$ & $\begin{array}{l}1.00 \\
1.00 \\
1.00\end{array}$ & $\begin{array}{c}0.97 \\
1.04 \\
1.01 \\
0 \\
\end{array}$ & $\begin{array}{l}1.05 \\
0.98 \\
1.02 \\
0.05 \\
\end{array}$ & $\begin{array}{l}1.00 \\
1.00 \\
1.00 \\
0.02 \\
\end{array}$ \\
\hline \multirow{2}{*}{ Cepharantin } & \multirow{2}{*}{$\begin{array}{l}0.05 \mathrm{mg} \\
\text { s.c. }\end{array}$} & $\mathrm{K}$-index & $\begin{array}{l}1 \\
2 \\
\text { Durchschn. } \\
\text { Differenz }\end{array}$ & $\begin{array}{l}60.0 \\
43.1 \\
50.3\end{array}$ & $\begin{array}{l}73.6 \\
53.9 \\
56.7 \\
-5.4 \\
\end{array}$ & $\begin{array}{r}77.8 \\
77.5 \\
77.8 \\
8.4 \\
\end{array}$ & $\begin{array}{r}67.9 \\
66.7 \\
66.8 \\
-1.0 \\
\end{array}$ \\
\hline & & P-zahl & $\begin{array}{c}1 \\
2 \\
\text { Durchschn. } \\
\text { Differenz }\end{array}$ & $\begin{array}{l}1.00 \\
1.00 \\
1.00\end{array}$ & $\begin{array}{l}1.17 \\
1.08 \\
1.13 \\
0.14\end{array}$ & $\begin{array}{l}1.10 \\
1.02 \\
1.06 \\
0.09\end{array}$ & $\begin{array}{l}1.04 \\
1.02 \\
1.03 \\
0.05\end{array}$ \\
\hline
\end{tabular}

Wenn man die Phagozytose der Leukozyten nach Sugiyama ${ }^{7)}$ bestimmt, so sieht man aus Tabelle VII, dass die Phagozytose der Leukozyten durch Penicillin Mix, K, Streptomycin oder Mapharsol in kleiner Menge auffallend gesteigert wird, während diese Wirkung durch Lumina 1 
TABELLE V

Einfluss der Antisyphilitika

\begin{tabular}{|c|c|c|c|c|c|c|c|}
\hline & \multirow{2}{*}{$\begin{array}{c}\text { Dose } \\
\text { (pro kg) }\end{array}$} & \multirow[t]{2}{*}{ 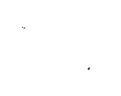 } & \multirow{2}{*}{$\begin{array}{l}\text { Nr. des } \\
\text { Kaninchens }\end{array}$} & \multirow{2}{*}{$\begin{array}{l}\text { Vor } \\
\text { Inj. }\end{array}$} & \multicolumn{3}{|c|}{ Zeit nach Injektion (St.) } \\
\hline & & & & & 1 & 3 & 5 \\
\hline \multirow{4}{*}{ Mapharse } & \multirow{2}{*}{$\begin{array}{c}5 \mathrm{mg} \\
\text { i.v. }\end{array}$} & K-index & $\begin{array}{l}1 \\
2 \\
\text { Durchschn. } \\
\text { Differenz }\end{array}$ & $\begin{array}{l}45.9 \\
41.5 \\
43.5\end{array}$ & $\begin{array}{l}50.0 \\
48.7 \\
49.3 \\
-8.0\end{array}$ & $\begin{array}{r}68.2 \\
54.5 \\
61.1 \\
4.9\end{array}$ & $\begin{array}{r}64.0 \\
58.3 \\
61.2 \\
7.5\end{array}$ \\
\hline & & P-zahl & $\begin{array}{l}1 \\
\stackrel{1}{2} \\
\text { Durchschn. } \\
\text { Differenz }\end{array}$ & $\begin{array}{l}1.00 \\
1.00 \\
1.00\end{array}$ & $\begin{array}{l}1.01 \\
1.00 \\
1.00 \\
0\end{array}$ & $\begin{array}{l}1.05 \\
1.01 \\
1.03 \\
0.04\end{array}$ & $\begin{array}{r}1.02 \\
1.00 \\
1.01 \\
-0.01\end{array}$ \\
\hline & \multirow{2}{*}{$\begin{array}{l}0.5 \mathrm{mg} \\
\text { i.v. }\end{array}$} & K-index & $\begin{array}{l}1 \\
\stackrel{1}{2} \\
\text { Durchschn. } \\
\text { Differenz }\end{array}$ & $\begin{array}{l}38.3 \\
36.8 \\
37.5\end{array}$ & $\begin{array}{r}37.3 \\
32.9 \\
34.6 \\
-16.7\end{array}$ & $\begin{array}{r}38.9 \\
33.8 \\
36.5 \\
-13.7\end{array}$ & $\begin{array}{l}42.6 \\
45.9 \\
44.3 \\
-5.4\end{array}$ \\
\hline & & P-zahl & \begin{tabular}{l}
1 \\
\multicolumn{2}{c}{} \\
Durchschn. \\
Differenz
\end{tabular} & $\begin{array}{l}1.00 \\
1.00 \\
1.00\end{array}$ & $\begin{array}{l}1.00 \\
1.02 \\
1.01 \\
0.01\end{array}$ & $\begin{array}{l}1.01 \\
1.05 \\
1.03 \\
0.04\end{array}$ & $\begin{array}{l}1.10 \\
1.09 \\
1.10 \\
0.08\end{array}$ \\
\hline \multirow{2}{*}{ Neosalvarsan } & \multirow{2}{*}{$\begin{array}{l}0.5 \mathrm{mg} \\
\text { i.v. }\end{array}$} & K-index & \begin{tabular}{l}
\multicolumn{1}{c}{} \\
$\stackrel{2}{2}$ \\
Durchschn. \\
Differenz
\end{tabular} & $\begin{array}{l}45.3 \\
41.2 \\
43.1\end{array}$ & $\begin{array}{l}51.7 \\
58.8 \\
55.3 \\
-1.6\end{array}$ & $\begin{array}{l}58.8 \\
50.0 \\
54.2 \\
-1.6\end{array}$ & $\begin{array}{r}63.1 \\
55.2 \\
58.8 \\
4.5\end{array}$ \\
\hline & & P-zahl & $\begin{array}{c}1 \\
2 \\
\text { Durchschn. } \\
\text { Differenz }\end{array}$ & $\begin{array}{l}1.00 \\
1.00 \\
1.00\end{array}$ & $\begin{array}{r}0.97 \\
1.00 \\
0.99 \\
-0.01\end{array}$ & $\begin{array}{r}0.98 \\
0.98 \\
0.98 \\
-0.01\end{array}$ & $\begin{array}{c}1.02 \\
1.01 \\
1.02 \\
0\end{array}$ \\
\hline
\end{tabular}

wieder völlig gehemmt wird.

Daraus wird klar, dass diese Antibiotika nicht nur die Funktion des RES, sondern auch diejenige der Leukozyten steigern.

\section{Diskussion}

Die Bedeutung des RES beim Wirkungsmechanismus der Chemotherapeutika ist bis heute von verschiedenen Autoren bestritten worden: einerseits berichten Kritschewsky, ${ }^{8)}$ Jungeblut $^{91}$ und Feld ${ }^{10}$ ) über eine dyschemotherapeutische Wirkung bei der Milzexstirpation und der reticuloendothelialen Blockade, anderseits betonten Kikuth ${ }^{11}$ und Prigge ${ }^{12)}$, dass dem sogenannten Reticuloendothel keine besondere Bedeutung für die Wirkung der Chemotherapeutika beigemessen werden kann. Weiterhin bemerkte Jansco, ${ }^{13)}$ dass die Wirkung der Chemotherapeutika sich mit der biologischen Schutzfunktion des Reticuloendothels kombiniert.

In Bezug auf den Einfluss der Sulfonamide berichtet $\mathrm{Oh}^{14)}$ über die Unbeeinflussbarkeit, während Morinka ${ }^{15}$ eine Vermehrung des Kon- 
TABELLE VI

Einfluss des Luminals auf die Wirkung der Chemotherapeutika

\begin{tabular}{|c|c|c|c|c|c|c|c|}
\hline & \multirow{2}{*}{$\begin{array}{c}\text { Dose } \\
\text { (pro kg) }\end{array}$} & \multirow[t]{2}{*}{, } & \multirow{2}{*}{$\begin{array}{l}\text { Nr. des } \\
\text { Kaninchens }\end{array}$} & \multirow{2}{*}{$\begin{array}{l}\text { Vor } \\
\text { Inj. }\end{array}$} & \multicolumn{3}{|c|}{ Zeit nach Injektion (St.) } \\
\hline & & & & & 1 & 3 & 5 \\
\hline \multirow{2}{*}{ Luminal } & \multirow{2}{*}{$\begin{array}{l}30 \mathrm{mg} \\
\text { i.m. }\end{array}$} & K-index & \begin{tabular}{l}
1 \\
\multicolumn{2}{c}{1} \\
Durchschn. \\
Differenz
\end{tabular} & $\begin{array}{l}50.0 \\
45.8 \\
67.8\end{array}$ & $\begin{array}{l}55.6 \\
70.6 \\
63.0 \\
13.0 \\
\end{array}$ & $\begin{array}{l}68.0 \\
66.7 \\
67.3 \\
15.2 \\
\end{array}$ & $\begin{array}{l}65.1 \\
65.0 \\
65.5 \\
14.0 \\
\end{array}$ \\
\hline & & P-zahl & \begin{tabular}{l}
\multicolumn{1}{c}{1} \\
2 \\
Durchschn. \\
Differenz
\end{tabular} & $\begin{array}{l}1.00 \\
1.00 \\
1.00\end{array}$ & $\begin{array}{c}1.00 \\
1.00 \\
1.00 \\
0\end{array}$ & $\begin{array}{l}1.00 \\
0.99 \\
1.00 \\
0.01\end{array}$ & $\begin{array}{r}0.96 \\
0.92 \\
0.94 \\
-0.07\end{array}$ \\
\hline \multirow{2}{*}{ Neogerison } & \multirow{2}{*}{$\begin{array}{l}100 \mathrm{mg} \\
\text { i.v. }\end{array}$} & $\mathrm{K}$-index & $\begin{array}{l}1 \\
2 \\
\text { Durchschn. } \\
\text { Differenz }\end{array}$ & $\begin{array}{l}58.2 \\
39.4 \\
49.1\end{array}$ & $\begin{array}{r}54.4 \\
44.3 \\
49.3 \\
-11.6 \\
\end{array}$ & $\begin{array}{r}72.4 \\
54.8 \\
64.7 \\
-3.5 \\
\end{array}$ & $\begin{array}{r}69.5 \\
43.3 \\
56.0 \\
-10.6 \\
\end{array}$ \\
\hline & & P-zahl & \begin{tabular}{l}
1 \\
\multicolumn{1}{c}{1} \\
Durchschn. \\
Differenz
\end{tabular} & $\begin{array}{l}1.00 \\
1.00 \\
1.00\end{array}$ & $\begin{array}{l}1.01 \\
1.09 \\
1.05 \\
0.06 \\
\end{array}$ & $\begin{array}{l}1.03 \\
1.07 \\
1.05 \\
0.08 \\
\end{array}$ & $\begin{array}{l}1.11 \\
1.04 \\
1.08 \\
0.10 \\
\end{array}$ \\
\hline \multirow{2}{*}{$\begin{array}{l}\text { Neogerison } \\
\text { nach Luminal }\end{array}$} & \multirow{2}{*}{$\begin{array}{c}\text { Neogerison } \\
100 \mathrm{mg} \\
\text { i.v. } \\
\text { Luminal } \\
30 \mathrm{mg} \\
\text { i.m. }\end{array}$} & $\mathrm{K}$-index & \begin{tabular}{l}
\multicolumn{1}{c}{1} \\
2 \\
Durchschn. \\
Differenz
\end{tabular} & $\begin{array}{l}32.3 \\
26.1 \\
29.5\end{array}$ & $\begin{array}{r}54.4 \\
41.1 \\
48.8 \\
5.5 \\
\end{array}$ & $\begin{array}{l}72.2 \\
58.5 \\
65.8 \\
23.6\end{array}$ & $\begin{array}{l}70.2 \\
65.0 \\
67.3 \\
27.2 \\
\end{array}$ \\
\hline & & P-zahl & \begin{tabular}{l}
\multicolumn{1}{c}{1} \\
2 \\
Durchschn. \\
Differenz \\
\end{tabular} & $\begin{array}{l}1.00 \\
1.00 \\
1.00\end{array}$ & $\begin{array}{c}1.02 \\
0.98 \\
1.00 \\
0 \\
\end{array}$ & $\begin{array}{l}1.04 \\
0.95 \\
1.00 \\
0.01 \\
\end{array}$ & $\begin{array}{r}1.02 \\
0.92 \\
1.02 \\
-0.05 \\
\end{array}$ \\
\hline \multirow{2}{*}{ Penicillin $\mathrm{K}$} & \multirow{2}{*}{$\begin{array}{c}20000 \mathrm{EH} \\
\text { i.m. }\end{array}$} & $\mathbf{K}$-index & \begin{tabular}{l}
\multicolumn{1}{c}{1} \\
2 \\
Durchschn. \\
Differenz \\
\end{tabular} & $\begin{array}{l}42.7 \\
28.9 \\
36.7\end{array}$ & $\begin{array}{r}45.0 \\
33.1 \\
39.9 \\
-10.6 \\
\end{array}$ & $\begin{array}{l}48.0 \\
39.0 \\
44.0 \\
-5.4 \\
\end{array}$ & $\begin{array}{l}66.7 \\
44.1 \\
57.2 \\
10.3 \\
\end{array}$ \\
\hline & & P-zahl & $\begin{array}{c}1 \\
2 \\
\text { Durchschn. } \\
\text { Differenz } \\
\end{array}$ & $\begin{array}{l}1.00 \\
1.00 \\
1.00\end{array}$ & $\begin{array}{l}1.01 \\
1.03 \\
1.02 \\
0.02 \\
\end{array}$ & $\begin{array}{l}1.06 \\
1.02 \\
1.04 \\
0.05 \\
\end{array}$ & $\begin{array}{r}0.99 \\
0.99 \\
0.99 \\
-0.03 \\
\end{array}$ \\
\hline \multirow{2}{*}{$\begin{array}{l}\text { Penicillin } \mathrm{K} \\
\text { nach Luminal }\end{array}$} & \multirow{2}{*}{$\begin{array}{c}\text { Penicillin K } \\
20000 \mathrm{EH} \\
\text { i.m. } \\
\text { Luminal } \\
30 \mathrm{mg} . \\
\text { i.m. }\end{array}$} & K-index & \begin{tabular}{l}
1 \\
\multicolumn{2}{c}{1} \\
Durchschn. \\
Differenz
\end{tabular} & $\begin{array}{l}43.0 \\
39.1 \\
41.1\end{array}$ & $\begin{array}{l}75.8 \\
51.2 \\
64.1 \\
18.5 \\
\end{array}$ & $\begin{array}{l}72.1 \\
71.3 \\
71.8 \\
19.7 \\
\end{array}$ & $\begin{array}{l}70.0 \\
68.0 \\
69.1 \\
10.5 \\
\end{array}$ \\
\hline & & P-zahI & \begin{tabular}{l}
\multicolumn{1}{c}{1} \\
\multicolumn{1}{c}{2} \\
Durchschn. \\
Differenz
\end{tabular} & $\begin{array}{l}1.00 \\
1.00 \\
1.00\end{array}$ & $\begin{array}{r}1.00 \\
0.91 \\
0.96 \\
-0.05 \\
\end{array}$ & $\begin{array}{l}1.13 \\
0.99 \\
1.06 \\
0.04\end{array}$ & $\begin{array}{l}1.13 \\
1.03 \\
1.08 \\
0.04\end{array}$ \\
\hline \multirow{2}{*}{ Streptomycin } & \multirow{2}{*}{$\begin{array}{c}20000 \mathrm{EH} \\
\text { i.m. }\end{array}$} & $\mathrm{K}$-index & \begin{tabular}{l}
\multicolumn{1}{c}{1} \\
2 \\
Durchschn. \\
Differenz \\
\end{tabular} & $\begin{array}{l}42.6 \\
41.9 \\
42.3\end{array}$ & $\begin{array}{l}45.4 \\
55.2 \\
50.0 \\
-6.1 \\
\end{array}$ & $\begin{array}{l}33.9 \\
52 \cdot 7 \\
45.3 \\
-9.4 \\
\end{array}$ & $\begin{array}{l}47.7 \\
57.0 \\
52.3 \\
-0.2 \\
\end{array}$ \\
\hline & & P-zahl & $\begin{array}{c}1 \\
2 \\
\text { Durchschn. } \\
\text { Differenz } \\
\end{array}$ & $\begin{array}{l}1.00 \\
1.00 \\
1.00\end{array}$ & $\begin{array}{l}1.01 \\
1.04 \\
1.03 \\
0.03 \\
\end{array}$ & $\begin{array}{l}1.05 \\
1.07 \\
1.06 \\
0.07 \\
\end{array}$ & $\begin{array}{l}1.09 \\
1.06 \\
1.08 \\
0.06 \\
\end{array}$ \\
\hline \multirow{2}{*}{$\begin{array}{l}\text { Streptomycin } \\
\text { nach Luminal }\end{array}$} & \multirow{2}{*}{$\begin{array}{c}\text { Streptomycin } \\
20000 \mathrm{EH} \\
\text { i.m. } \\
\text { Luminal } \\
30 \mathrm{mg} \\
\text { i.m. }\end{array}$} & $\mathrm{K}$-index & \begin{tabular}{l}
\multicolumn{1}{c}{1} \\
2 \\
Durchschn. \\
Differenz \\
\end{tabular} & $\begin{array}{l}37.3 \\
34.4 \\
35.7\end{array}$ & $\begin{array}{l}48.0 \\
50.0 \\
49.1 \\
-0.4 \\
\end{array}$ & $\begin{array}{l}62.0 \\
60.8 \\
61.2 \\
12.8 \\
\end{array}$ & $\begin{array}{l}80.0 \\
63.1 \\
71.5 \\
25.6 \\
\end{array}$ \\
\hline & & P-zahl & \begin{tabular}{l}
\multicolumn{1}{c}{1} \\
2 \\
Durchschn. \\
Differenz
\end{tabular} & $\begin{array}{l}1.00 \\
1.00 \\
1.00\end{array}$ & $\begin{array}{l}1.02 \\
0.99 \\
1.01 \\
0.01\end{array}$ & $\begin{array}{l}1.08 \\
1.03 \\
1.06 \\
0.07\end{array}$ & $\begin{array}{l}1.05 \\
1.04 \\
1.05 \\
0.03\end{array}$ \\
\hline
\end{tabular}


TABELLE VII

Einfluss der Chemotherapeutika auf die Phagozytose der Leukozyten

\begin{tabular}{|c|c|c|c|c|c|c|c|c|c|}
\hline & \multirow{2}{*}{$\begin{array}{c}\text { Dose } \\
\text { (pro kg) }\end{array}$} & & \multirow{2}{*}{$\begin{array}{l}\text { Vor } \\
\text { Inj. }\end{array}$} & \multicolumn{6}{|c|}{ Zeit nach Injektion (St.) } \\
\hline & & & & 1 & 2 & 3 & 4 & 5 & 6 \\
\hline Kontrolle & & $\begin{array}{l}\text { Phagozytose } \\
\text { Differenz }\end{array}$ & 1.46 & $\begin{array}{r}1.41 \\
-0.05\end{array}$ & $\begin{array}{r}1.23 \\
-0.23\end{array}$ & $\begin{array}{r}1.25 \\
-0.21\end{array}$ & $\begin{array}{r}1.19 \\
-0.27\end{array}$ & $\begin{array}{r}1.40 \\
-0.06\end{array}$ & $\begin{array}{r}1.42 \\
-0.04\end{array}$ \\
\hline Penicillin Mix & $\begin{array}{c}20000 \mathrm{EH} \\
\text { i.m. }\end{array}$ & $\begin{array}{l}\text { Phagozytose } \\
\text { Differenz }\end{array}$ & 1.90 & $\begin{array}{r}1.77 \\
-0.06\end{array}$ & & $\begin{array}{l}2.07 \\
0.40\end{array}$ & & $\begin{array}{l}1.95 \\
0.13\end{array}$ & \\
\hline Penicillin $\mathrm{K}$ & $\begin{array}{l}20000 \mathrm{EH} \\
\text { i.m. }\end{array}$ & $\begin{array}{l}\text { Phagyzytose } \\
\text { Differenz }\end{array}$ & 1.27 & $\begin{array}{l}1.49 \\
0.27\end{array}$ & $\begin{array}{r}0.69 \\
-0.35\end{array}$ & $\begin{array}{l}1.25 \\
0.19\end{array}$ & $\begin{array}{l}1.23 \\
0.23\end{array}$ & $\begin{array}{r}1.14 \\
-0.07\end{array}$ & $\begin{array}{r}1.15 \\
-0.08\end{array}$ \\
\hline Streptomycin & $\begin{array}{l}20000 \mathrm{EH} \\
\text { i.m. }\end{array}$ & $\begin{array}{l}\text { Phagozytose } \\
\text { Differenz }\end{array}$ & 1.97 & $\begin{array}{l}1.93 \\
0.03\end{array}$ & $\begin{array}{l}2.03 \\
0.31\end{array}$ & $\begin{array}{c}1.74 \\
0\end{array}$ & $\begin{array}{l}1.99 \\
0.31\end{array}$ & $\begin{array}{l}2.04 \\
0.15\end{array}$ & $\begin{array}{l}2.00 \\
0.03\end{array}$ \\
\hline \multirow{2}{*}{ Mapharsol } & $\begin{array}{l}5 \mathrm{mg} \\
\text { i.m. }\end{array}$ & $\begin{array}{l}\text { Phagozytose } \\
\text { Differenz }\end{array}$ & 2.20 & $\begin{array}{r}1.63 \\
-0.52\end{array}$ & $\begin{array}{r}1.11 \\
-0.86\end{array}$ & & $\begin{array}{r}0.77 \\
-1.16\end{array}$ & $\begin{array}{r}0.52 \\
-1.62\end{array}$ & $\begin{array}{r}0.71 \\
-1.45\end{array}$ \\
\hline & $\begin{array}{c}0.5 \mathrm{mg} \\
\text { i.v. }\end{array}$ & $\begin{array}{l}\text { Phagozytose } \\
\text { Differenz }\end{array}$ & 1.11 & $\begin{array}{l}1.26 \\
0.20\end{array}$ & $\begin{array}{l}1.20 \\
0.32\end{array}$ & $\begin{array}{l}0.94 \\
0.04\end{array}$ & $\begin{array}{r}0.78 \\
-0.06\end{array}$ & $\begin{array}{r}0.43 \\
-0.62\end{array}$ & $\begin{array}{r}0.33 \\
-0.74\end{array}$ \\
\hline $\begin{array}{l}\text { Streptomycin } \\
\text { nach Luminal }\end{array}$ & $\begin{array}{c}\text { Streptomycin } \\
20000 \mathrm{EH} \\
\text { i.m. } \\
\text { Luminal } \\
30 \mathrm{mg} \\
\text { i.m. }\end{array}$ & $\begin{array}{l}\text { Phagozytose } \\
\text { Differenz }\end{array}$ & 1.66 & $\begin{array}{r}1.52 \\
-0.04\end{array}$ & $\begin{array}{r}1.07 \\
-0.36\end{array}$ & & $\begin{array}{r}1.12 \\
-0.27\end{array}$ & & $\begin{array}{l}1.25 \\
0.37\end{array}$ \\
\hline
\end{tabular}

gorotindex bemerkt und Mizuta ${ }^{16)}$ eine Steigerung der Farbstoffausscheidungsfunktion der Leber beobachtet. Weiterhin betonen Finkelstein, ${ }^{16}$ Neufeld ${ }^{18)}$ und Tomizuka ${ }^{19)}$ eine Steigerung der Phagozytose der Leukozyten durch Sulfonamide, doch negieren Sandkühler, ${ }^{201}$ Kubo usw. ${ }^{21}$ diese Wirkung.

Hinsichtlich der Wirkung der Antibiotika berichtet Sakata, ${ }^{22)}$ dass Streptomycin die Funktion des RES bei Tuberkulosekranken steigert. Weiterhin betonen Tomizuka, ${ }^{19)}$ Kimura $^{231}$ und Harakawa ${ }^{24)}$ eine Vermehrung der Phagozytose sowie der Wanderungsgeschwindigkeit der Leukozyten durch Streptomycin. Vor allem bemerken Kubo usw. ${ }^{21)}$ dass nicht nur Streptomycin, sondern auch Penicillin die Phagozytose der Leukozyten steigern, während Sandkühler ${ }^{20)}$ eine solche Wirkung von Penicillin negiert.

Betreffs der antisyphilitischen Arsenpräparate bemerken Goldzieher und Peck ${ }^{25}$ ) eine Steigerung der Funktion des RES, aber Haendel und Malet ${ }^{26)}$ eine Herabsetzung derselben. Weiterhin betont Iwashita ${ }^{27)}$ eine Verminderung der Funktion des RES bei grosser Dose und eine Vermehrung derselben bei kleiner Dose.

Aus unseren Versuchsergebnissen ist ersichtlich, dass verschiedene Sulfonamide und Antibiotika, mit Einschluss von antituberkulösn sowie antisyphilitischen Mitteln, die Funktion des RES sowie die Phagozytose der Leukozyten steigern, dagegen ist ihr Einfluss auf die Leberzellenfunktion 
nicht einheitlich. Sogar diese Wirkung der Sulfonamide und Antibiotika wird durch Luminal ganz aufgehoben. Deshalb ist zu vermuten, dass diese Sulfonamide und Antibiotika vermittelst des vegetativen Nervenzentrums, in ähnlicher Weise wie bei Vitamin, die Funktion des RES stimulieren.

Infolgedessen ist es nicht zu leugnen, dass sich die Funktion des RES beim Wirkungsmechanismus der Sulfonamide sowie der Antibiotika, mit Einschluss von antituberkulösen Mitteln, beteiligt.

\section{ZUSAMMENFASSUNG}

Wenn wir unsere Versuchsergebnisse zusammenfassen, so können wir folgenderweise schliessen :

1. Von den verschiedenen Sulfonamiden rufen Aktiweis, Trianon Theradiazine, Romezin, Sulzol, Sulzol-s, Neodiseptal Neogerison, Typhogerison, Protomin, Thiasin und Domian, nicht aber Homosulfamin, eine Verminderung des Kongorotindex hervor, dagegen steigt die Prothrombinzahl nur durch Aktiweis, Trianon, Neogerison, Typhogerison, Homosulfamin und Domian.

2. Von den verschiedenen Antibiotika setzen Penicillin G, Mix, K, wässriges Penicillin G, Streptomycin und Colistin, nicht aber Penicillin $\mathrm{K}$ in kleiner Menge und öligem Penicillin $\mathrm{G}$, den Kongorotindex herab. Dabei wird die Prothrombinzahl durch Penicillin G, Mix, K und Streptomycin gesteigert, sonst aber bleibt sie unverändert.

3. Verschiedene Tuberkulostatika wie PAS, Tibione und Cepharantin setzen den Kongorotindex nur bei geeigneter Menge herab, aber die Prothrombinzahl bleibt dabei fast unverändert, mit Ausnahme von Cepharantin.

4. Mapharsol, das antisyphilitische Arsenpräparat, setzt den Kongorotindex nur bei kleiner Dose herab und erhöht ihn bei grosser Dose, während Neoneonatvarsan auch in kleiner Menge eine sehr geringe Verminderung des Kongorotindex verursacht.

5. Die oben erwähnte Herabsetzung des Kongorotindex bei Neogerison, Penicillin $\mathrm{K}$ und Streptomycin wird durch Luminal völlig gehemmt.

6. Verschiedene Antibiotika wie Penicillin Mix, K und Streptomycin steigern die Phagozytose der Leukozyten, aber diese Wirkung wird durch Luminal wieder völlig gehemmt.

7. Daraus ist ersichtlich, dass diese Sulfonamide und Antibiotika die Funktion des RES und der Leukozyten vermittelst des vegetativen Nervenzentrums steigern. 


\section{Literatur}

1) Ehrlich, Berl. klin. Wschr., 1904, 329 ; Verh. Dtsch. Kongr. inn. Med., 1910, 27, 226.

2) Domagk, Dtsch. med. Wschr., 1935, 250.

3) Fleming, Brit. J. Exper. Med., 1929, 10, 226.

4) Waksman et al., Proc. Soc. Exper. Biol. Med., 1944, 35, 220.

5) Yamagata et al., J. Jap. Soc. Int. Med. (Jap.), 1952, 41, 338, 341 ; Tohoku J. Exper. Med., 1954, 59, 257.

6) Hasegawa, Studien über Chemotherapie der Tuberkulose (Jap.), 1942.

7) Sugiyama, Neue Studien und Methodik über Blut und Gewebe (Jap.), 1942.

8) Kritschewsky \& Meersohn, Zschr. Immunit. forsch., 1926, 45, 407.

9) Jungeblut, Zschr. Hyg., 1927, 107, 357.

10) Feld \& Schott, Ebenda, 1927, 107, 453.

11) Kikuth \& Regendanz, Zschr. Immunit. forsch., 1929, 61, 422.

12) Prigge, Med. Klin., 1931, 27, 1000.

13) Jansco, Zschr. exper. Med., 1929, 65, 98.

14) Oh, J. Med. Assoc. Taiwan (Jap.), 1944, 43, 397.

15) Morioka, Keio Igaku (Jap.), 1944, 24, 217.

16) Mizuta, J. Jap. Soc. Int. Med. (Jap.), 1951, 40, Nr. 3, 124.

17) Finkelstein \& Birkland, Science, 1938, 87, 441.

18) Neufeld \& Bär, Zschr. Hyg., 1940, 123, 116.

19) Tomizuka et al., Acta Haem. (Jap.), 1949, 13, Nr. 4, 253.

20) Sandkühler, Sang, 1950, 21, 400.

21) Kubo et al., Acta Haem. Jap. (Jap.), 1949, 13, Nr. 4, 254.

22) Sakata, Iryo (Jap.), 1949, 4, Nr. 2, 83.

23) Kimura, Acta Haem. Jap. (Jap.), 1949, 13, Nr. 4, 202.

24) Harakawa, Ebenda, 1949, 13, Nr. 4, 203.

25) Goldzieher \& Peck, Arch. Path., 1927, 3, 635.

26) Haendel \& Malet, Zschr. exper. Med., 1929, 67, 315.

27) Iwashita, Jap. J. Dermat. (Jap.), 1937, 41, 661, 813. 